\title{
Comparison between KPVT/SPM and SoHO/MDI magnetograms with an application to solar irradiance reconstructions
}

\author{
T. Wenzler ${ }^{1}$, S. K. Solanki ${ }^{2}$, N. A. Krivova ${ }^{2}$, and D. M. Fluri ${ }^{1}$ \\ ${ }^{1}$ Institute of Astronomy, ETH Zentrum, 8092 Zurich, Switzerland \\ e-mail: wenzler@astro.phys.ethz.ch \\ 2 Max-Planck-Institut für Sonnensystemforschung ${ }^{\star}, 37191$ Katlenburg-Lindau, Germany \\ e-mail: solanki@linmpi.mpg.de
}

Received 18 May 2004 / Accepted 23 July 2004

\begin{abstract}
To be able to use both space- and ground-based solar magnetograms and construct long time series of derived parameters it is important to cross-calibrate them so that we can estimate their reliability and combine them. Using two different techniques, we compare magnetograms as well as continuum images recorded by the Spectropolarimeter (SPM) on Kitt Peak and the Michelson Doppler Interferometer (MDI) on board SoHO. We find that the result obtained depends on the method used. The method we favour gives almost identical umbral and penumbral areas and very similar total magnetic fluxes in faculae. The magnetic fluxes in umbrae and penumbrae returned by the two instruments, however, differ considerably. We also demonstrate that SPM data can be employed to reconstruct total solar irradiance variations with almost the same accuracy as recently shown for MDI data.
\end{abstract}

Key words. Sun: solar-terrestrial relations - methods: data analysis - Sun: activity - Sun: faculae, plages Sun: magnetic fields - Sun: sunspots

\section{Introduction}

High resolution full-disk magnetograms obtained at regular intervals are an extremely valuable resource for solar cycle and other studies, including investigations of solar irradiance variations. Since the launch of the Solar and Heliospheric Observatory (SoHO) two such records exist. The Solar Oscillations Investigation/Michelson Doppler Imager (SOI/MDI) on board the ESA-NASA satellite SoHO has provided high quality magnetograms since 1996, while the Kitt Peak Vacuum Tower (KPVT) of the National Solar Observatory (NSO) has provided magnetograms since 1974 , although with reduced quality up to 1992 , when the Spectromagnetograph (SPM) became operational. Magnetograms at lower resolution are regularly recorded at the Wilcox Solar Observatory and the Mount Wilson Observatory. It is of considerable importance to make a careful comparison of the magnetograms obtained by MDI with those by SPM. This is especially important for reconstructing the total solar irradiance (TSI) back to 1974 , i.e. even further back than the start of space-based TSI observations in 1978. This would help to resolve the debate about any long-term drift in the composite data sets obtained from the various TSI measurements.

A central question for understanding solar variability is to what extent solar surface magnetism determines solar

\footnotetext{
^ Previously Max-Planck-Institut für Aeronomie.
}

irradiance variations. Following initial studies that used proxies of the magnetic field (see Foukal 1992, for an overview), more recent approaches to answering this question use the observed surface distribution of the solar magnetic field to reconstruct the irradiance (Chapman et al. 1996; Fligge et al. 2000a,b; Krivova et al. 2003). This approach has successfully reproduced with high accuracy the total and spectral irradiance measured by the Variability of solar IRradiance and Gravity Oscillations (VIRGO) experiment on SoHO, but has so far been restricted to MDI data, available only since 1996, i.e. for less than a solar cycle. For many purposes a longer time series, such as that provided by the KPVT magnetograms would be useful.

The general aim of the current paper is to critically and carefully compare the magnetic fluxes and flux distributions from the two data sets and thus to determine conversion factors between the two. A more immediate and particular aim is to demonstrate that reconstructions of total solar irradiance from 1996 to 2001 based on NASA/NSO SPM data are of almost the same quality as those made by Krivova et al. (2003), employing SoHO/MDI data. Besides comparing the magnetograms we also compare the continuum images and in particular the sunspots visible in them, since these data are also important for irradiance reconstructions.

In Sect. 2 we present the data sets used. In Sect. 3 we compare magnetograms and continuum intensity images taken with the NASA/NSO SPM at the KPVT and MDI on board SoHO. 


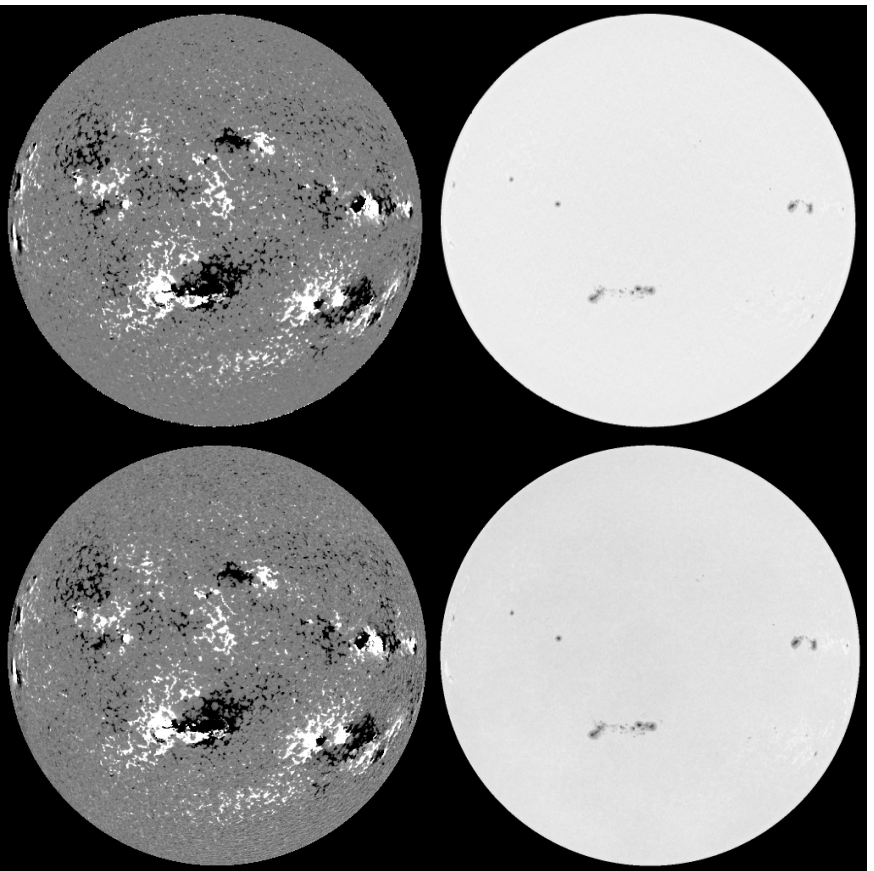

Fig. 1. Examples of magnetograms (left) and continuum intensity images after removal of limb-darkening (right) for November 10, 2001. The magnetogram and the corresponding continuum intensity image in the upper row were recorded by SPM, those in the lower row by MDI. The MDI magnetogram is a 20-min average.

We describe the model of total solar irradiance in Sect. 4. Our results of the reconstruction of total solar irradiance variations in cycle 23 based on KPVT/SPM data are summarized in Sect. 5. Finally, our conclusions and a summary are given in Sect. 6.

\section{Data}

\subsection{Description of the data}

The results discussed here are based on four data sets, namely data from SPM, MDI and VIRGO as well as the composite data of TSI from PMOD/WRC, Davos, Switzerland. The first set (henceforth referred to as SPM) is the set of daily full-disk magnetograms and corresponding continuum intensity images from the NASA/NSO SPM (Jones et al. 1992) at the KPVT derived from long-slit spectral polarimetry of the Fe I $8688 \AA$ spectral line. The SPM instrument gives an image of the Sun with $1788 \times 1788$ pixels. The full-disk images have a field of view of $34^{\prime} \times 34^{\prime}$ and a pixel size of $1.14^{\prime \prime} \times 1.14^{\prime \prime}$. The entire disk is scanned in about one hour. Data for 1475 days from 1996 January 1 to 2001 December 22 are available. A sample SPM magnetogram and the corresponding continuum intensity image recorded on 10th November 2001 are shown in the upper row of Fig. 1.

The SPM data (both the magnetograms and continuum intensity images) occasionally exhibit artifacts introduced by incomplete coverage of the solar disk, clouds, seeing and/or instrumental problems. If not identified and removed, magnetograms with artifacts would introduce a bias into the magnetic fluxes deduced from the SPM data and lead to an inaccurate

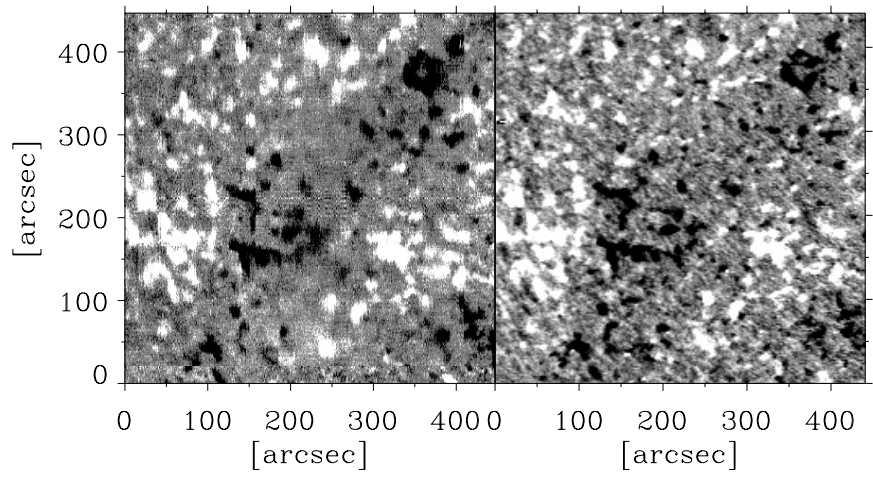

Fig. 2. Example of a region affected by clouds in an SPM magnetogram recorded on April 13, 1999 (left). The same region in the corresponding MDI magnetogram (right).

relation between SPM and MDI fluxes. They would introduce scatter into irradiance reconstructions and reduce the accuracy of other applications of these magnetograms. Therefore, we have carefully examined the magnetograms and the appropriate intensity images for these possible artifacts on all of these 1475 days. As an example we show in Fig. 2, in a region of the Sun with a field of view of about $450^{\prime \prime} \times 450^{\prime \prime}$, the negative influence of clouds on the quality of the SPM magnetogram (left image) recorded on April 13, 1999. As comparison we also plot the appropriate region of the MDI magnetogram recorded nearest in time (right image). In this case the SPM data miss a significant amount of magnetic flux. Such artifacts were flagged if the SPM magnetogram appeared fuzzy in places and the network at the affected locations was missing or smeared in the facular masks (see Sect. 3.2 for a description).

A further example (Fig. 3) shows the effect of an instrumental artifact in a region of the Sun with a field of view of about $450^{\prime \prime} \times 450^{\prime \prime}$. The quality loss in the SPM magnetogram (left image) recorded on April 22, 1999 as opposed to the corresponding region of the MDI magnetogram (right image) is well recognizable. In this case extra signal is introduced into the SPM. Such artifacts were flagged if the magnetogram appeared particularly noisy, which caused many single pixels to appear above the threshold in the facular masks. These masks became then so noisy that the network was difficult to identify.

We identified regions with artifacts or obvious loss of data quality (often also missing parts of the image, when a scan was not completed) on 314 days, constituting $21 \%$ of the original data record. Thus data for 1161 days from 1996 January 1 to 2001 December 22 can be used for irradiance reconstructions or other investigations that require complete magnetograms of uniform quality. We stress that magnetograms were not removed on the basis of the irradiance reconstructions and its agreement or disagreement with the observations.

The second data set employed here (henceforth referred to as MDI) is a set of daily full-disk magnetograms and continuum intensity images recorded by the SOI/MDI instrument on board the SoHO spacecraft. This instrument images the Sun on a $1024 \times 1024$ CCD camera, and records opposite states of polarization at different wavelengths in the Ni I $6768 \AA$ line, producing a magnetogram at a maximum cadence of about one 


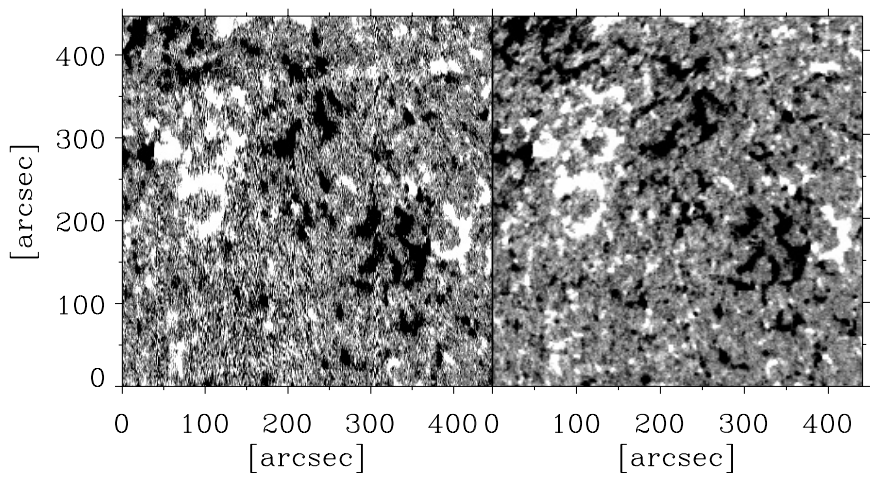

Fig. 3. Example of an SPM magnetogram with artifacts caused by instrumental problems (left) and the corresponding MDI magnetogram (right) for April 22, 1999.

per minute. The full-disk measurements have a field of view of $34^{\prime} \times 34^{\prime}$ and a pixel size of $2^{\prime \prime} \times 2^{\prime \prime}$. Data for 1495 days from 1996 May 19 to 2002 April 17 are considered here. The SOI/MDI instrument has been described in detail by Scherrer et al. (1995). The two images in the lower row of Fig. 1 show a magnetogram (averaged over 20 single magnetograms, taken at a cadence of 1 per minute) and the corresponding intensity image recorded by MDI on November 10, 2001. For the intensity images we always use single MDI frames, while for the magnetograms we consider averages over varying numbers of individual magnetograms.

Total solar irradiance reconstructions based on SPM and MDI data are compared with the TSI measurements obtained by the VIRGO instrument (Fröhlich et al. 1995) on board the SoHO spacecraft. We use the newest level 2 VIRGO data (Fröhlich \& Finsterle 2001) from 1996 February 7 to 2003 January 15 (Fröhlich 2003).

During the 3-month gap in SoHO's operation in summer 1998, as well as during a few other minor gaps at the end of 1998 and the beginning of 1999, we employed the composite record of TSI compiled from measurements by five independent space-based radiometers from 1978 through the present (Fröhlich \& Lean 1998; Fröhlich 2000; Fröhlich 2003). For this study we use version 25 of the composite measure of TSI from PMOD/WRC. During the considered intervals the composite basically consists of shifted ACRIM II data (Willson 1994).

\subsection{Noise level}

The noise level of the MDI magnetograms was determined by Ortiz et al. (2002). They applied an iterative technique to ensure that, to the extent possible, the magnetic signal is properly removed and is not counted as noise (but see Krivova \& Solanki 2004, for estimates of the magnetogram signal hidden in the noise). Ortiz et al. (2002) provided a 2D representation of the noise, which was found to vary significantly over the field of view. The average magnetic noise level for 5-min integrated MDI magnetograms is about $\sigma_{\text {mag, MDI }}=9 \mathrm{G}$, with the noise level varying roughly as $1 / \sqrt{T}$, where $T$ is the integration time in minutes of the employed MDI magnetogram,

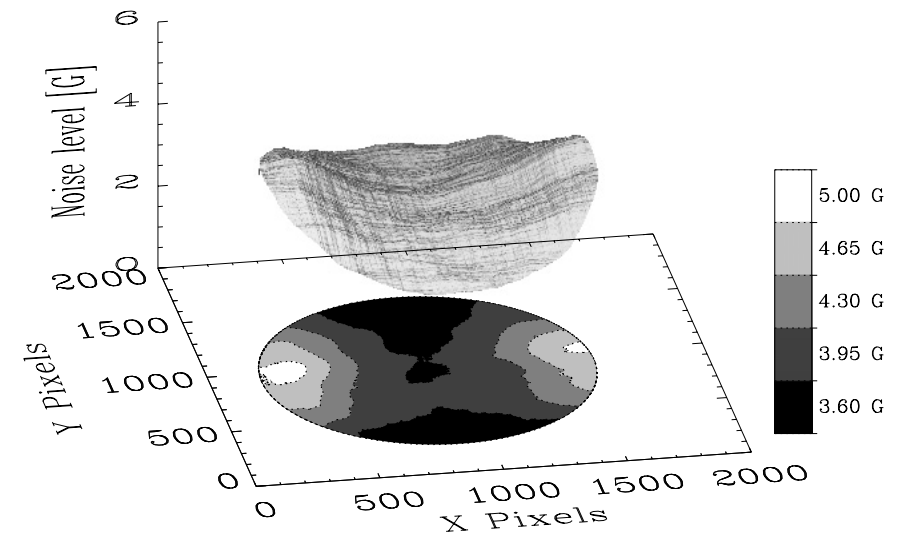

Fig. 4. Noise level (in G) of the SPM magnetograms as a function of location on the solar disk. Plotted is an average over the noise level surfaces of 14 magnetograms. The mean value of the surface is $4.2 \mathrm{G}$, with maximum and minimum values of about 5.1 and $3.6 \mathrm{G}$, respectively. Both the surface and the contours represent the standard deviation of the magnetogram signal (see text for details). The $y$-axis gives the direction from south to north, the $x$-axis from east to west.

or equivalently, the number of 1-min magnetograms averaged together. This is in good agreement with the value given by Scherrer et al. (1995).

We now determine the noise level of the SPM magnetograms, applying the same method as Ortiz et al. (2002). We use 14 low activity magnetograms from the year 1996, in order to avoid artifacts introduced by the presence of active regions. We calculate the standard deviation for the magnetic signal using a running $150 \times 150$ pixel box over the solar disk, but excluding an outer ring of 125 pixels width to avoid the limb. Outliers are replaced by the averages from nearby pixels, under the assumption that the outliers are affected by the magnetic field. This process is applied several times to eliminate any possible remaining influence of the magnetic field. In general the noise level converges to nearly the final value after only the second iteration. The resulting noise levels $\sigma_{\mathrm{mag} \text {, SPM }}$ for the selected low activity magnetograms are more or less constant over the solar disk but vary between different magnetograms. The values of the mean noise level ("mean" referring to the average over one single magnetogram) range from $3.3 \mathrm{G}$ to $5.9 \mathrm{G}$ with an average over all the studied magnetograms of $4.2 \mathrm{G}$. In Fig. 4 we show the calculated standard deviation surface averaged over all 14 days. Since the averaged noise level reaches values of slightly above $5 \mathrm{G}$ near the limb, we propose a conservative noise level of $5 \mathrm{G}$. Jones et al. (1992) and Jones et al. (2003) also found a noise level of the SPM magnetograms of about $5 \mathrm{G}$. The noise level in the SPM magnetograms is thus similar to the noise level in 20-min integrated MDI magnetograms. We note, however, that the noise does not entirely follow a Gaussian distribution, since even when taking a cutoff of $3 \sigma$ a significant number of points show a signal that is not spatially distributed like the magnetic network. Due to the scatter in the noise levels derived for the different days we cannot rule out a time dependence of the noise. Nonetheless, for simplicity we assume that the noise is time-independent in the rest of this paper. Since for the remaining analysis the MDI noise 
level, which is generally higher, is critical, we do not expect this assumption to affect the results of our work.

\section{Comparison of KPVT/SPM and SoHO/MDI data}

In this section we compare magnetograms and continuum intensity images taken with the two different instruments NASA/NSO SPM at the KPVT and the SoHO/MDI. We have selected 24 days with different levels of activity between 1997 and 2001 for the detailed comparison. In Sect. 3.1 we use a similar comparison method as Jones \& Ceja (2001) and Thornton \& Jones (2002). Partly this is done to check for consistency with their findings, although we also refine a part of this analysis compared to theirs. This method is, however, not optimal since it is affected by the amount of noise in the data and by the sunspots, whose magnetic signals are clearly different in the two data sets. In particular, the MDI data provide far greater values of the line-of-sight (LOS) component, $B_{\mathrm{LOS}}$, of the magnetic field in sunspot umbrae (see Sect. 3.3). Therefore we also use an alternative comparison method described in Sect. 3.2.

The noise level in the SPM magnetograms is roughly a factor of 4-5 lower than in the original MDI magnetograms recorded at 1-min cadence. In the following data comparison (Sects. 3.1 and 3.2) we therefore also employ averages over 5, 20 and 56 single MDI magnetograms to reduce the noise level. The individual magnetograms were rotated to the middle of the 5,20 or $56 \mathrm{~min}$ integration time before averaging, to compensate for solar rotation. Care has been taken to use MDI magnetograms and intensity images obtained as close in time to the SPM magnetograms as possible. For all SPM magnetograms, the MDI magnetograms were recorded during the time that the SPM was scanning the solar disk. The average time difference between the MDI intensities and magnetograms was about $30 \mathrm{~min}$. The MDI intensity images were then rotated to the observation time of the magnetograms (i.e. to the observation time of the 1-min magnetograms or to the middle of the 5, 20 ore 56 min integration time of the corresponding averaged magnetograms). Finally, the SPM and MDI intensity images were corrected for limb-darkening effects following Neckel \& Labs (1994).

\subsection{Histogram comparison}

We compare all the pairs of full-disk magnetograms for each of the selected 24 days employing a method earlier used by Jones et al. (2000) and Jones \& Ceja (2001). Following these authors we refer to it as histogram equating. It is independent of the different pixel sizes of the two types of magnetograms. The basic underlying assumption of this method is that SPM and MDI magnetograms differ only in the scale of the magnetic field. By comparing the relative number of pixels (as opposed to absolute numbers) with a certain magnetic field the two data sets become directly comparable despite the different pixel size.

To obtain these comparable data sets we form histograms of the magnetic field distribution in a special way. No magnetic threshold is applied, i.e. all magnetic pixels that lie within the solar disk are considered. For each magnetogram all

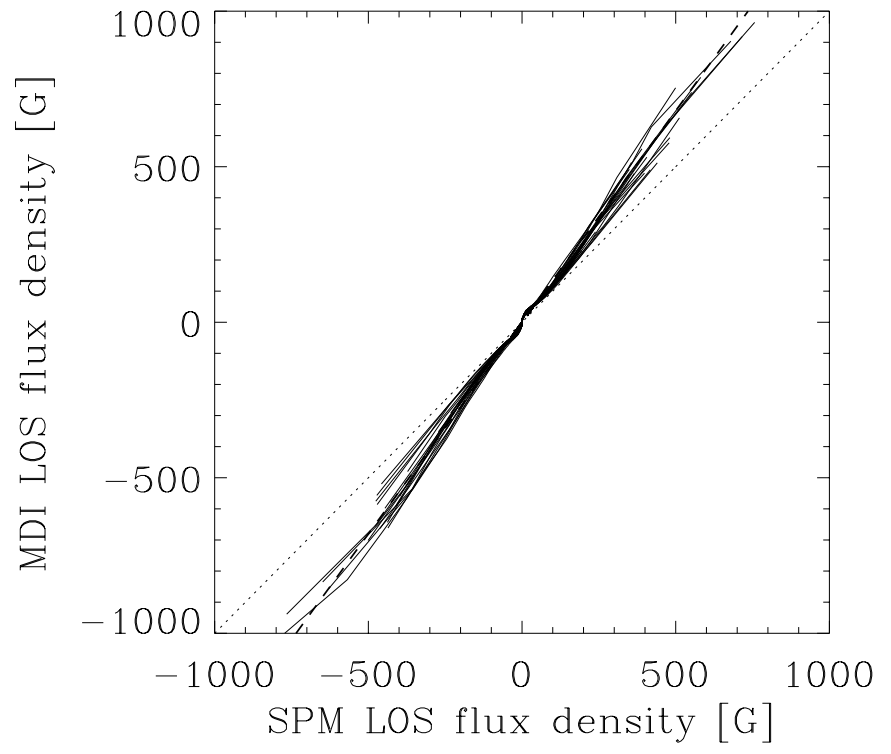

Fig. 5. Histogram-equating curves for 24 individual days between 1997 and 2001, calculated with SPM magnetograms and standard 1-min MDI magnetograms. The dotted diagonal line represents the expectation values for identical SPM and MDI magnetograms, the dashed line a linear regression.

pixels within the solar disk are divided up into different bins as follows: pixels with a positive line-of-sight (LOS) component $B_{\mathrm{LOS}}$ of the magnetic field are put into 1000 equally populated bins with increasing $B_{\mathrm{LOS}}$ value. In other words, if the pixels are sorted with increasing $B_{\mathrm{LOS}}$ and defining $N$ as one thousandth of the total number of pixels, then the first $N$ pixels (with positive $B_{\mathrm{LOS}}$ ) are put into the first bin, the second $N$ pixels into the second bin and so on. The same division into 1000 bins is performed for pixels with negative $B_{\mathrm{LOS}}$. Then, to each of these bins the average magnetic field $B_{\mathrm{LOS}}$ as obtained from all of its pixels is assigned. This set of $B_{\mathrm{LOS}}$ values for the different bins forms the "equating" table of a magnetogram. These equating tables from the SPM and MDI data sets are the relative magnetic field distributions, which allow a direct comparison of SPM and MDI magnetograms.

In Fig. 5 the MDI equating tables based on standard 1-min magnetograms are plotted against the SPM equating tables for the 24 individual days between 1997 and 2001. A linear fit to the 24 histogram-equating curves has been computed. The resulting slope and intercept are $1.360 \pm 0.006$ and $-0.261 \pm 0.149$, respectively (see Table 1 ). The error bars are the formal errors of the regression. Thornton \& Jones (2002) used a similar histogram-equating method, which they also applied to the original 1-min MDI magnetograms. Their resulting slope and intercept of the MDI vs. SPM average of linear fits to histogram-equating curves between 1996 and 2001 are $1.362 \pm 0.035$ and $3.837 \pm 1.866$, respectively (private communication by H. P. Jones, see also Jones \& Ceja 2001). The slopes obtained in the two studies coincide within the $1 \sigma$ error bars and are greater than unity, i.e. MDI shows larger magnetic fluxes than SPM. As can be seen from Fig. 5, there is considerable scatter from one day to the other so that the exact choice of dates is relevant. Details such as the limiting 
Table 1. Average of linear fits to histogram-equating curves for 1,5, 20 and 56 min integrated MDI magnetograms.

\begin{tabular}{ccc}
\hline \hline Comparison & Intercept & Slope \\
\hline 1-min MDI vs. SPM & $-0.261 \pm 0.149$ & $1.360 \pm 0.006$ \\
5-min MDI vs. SPM & $-0.113 \pm 0.067$ & $1.268 \pm 0.003$ \\
20-min MDI vs. SPM & $-0.118 \pm 0.051$ & $1.226 \pm 0.001$ \\
56-min MDI vs. SPM & $-0.116 \pm 0.051$ & $1.212 \pm 0.002$ \\
\hline
\end{tabular}

Results of Thornton \& Jones (2002):

1-min MDI vs. SPM $\quad 3.837 \pm 1.866 \quad 1.362 \pm 0.035$

$\mu$ values $(\mu=\cos (\theta)$, where $\theta$ is the heliocentric angle) also play a role, especially since MDI data suffer from very strong noise at some locations right at the limb. Nonetheless, we can confirm the results of Thornton \& Jones (2002).

Figure 6 illustrates the influence of the integration time of MDI magnetograms on the MDI vs. SPM histogram-equating curves for November 10, 2001. Plotted are comparisons of histogram-equating curves for 1, 5, 20 and 56 min integrated MDI magnetograms, corresponding to increasingly lower noise levels. A restricted flux density range around the origin is shown to illustrate the influence of the different noise levels more clearly. Note in particular the large difference between the 1-min MDI and the other curves. The differences persist well beyond the $1 \sigma$ noise level. The slopes of the linear fits to the histogram-equating curves for the MDI magnetograms based on different amounts of averaging are given in Table 1. We see that the slopes decrease with decreasing $1 \sigma$ noise levels, i.e. with increasing integration time.

A part, if not all, of the temporal variability suggested by Fig. 5 may be due to variable seeing. Krivova $\&$ Solanki (2004) have demonstrated just how sensitive the total amount of magnetic flux detected by a magnetogram is to spatial resolution. Variable seeing from one day to the next can therefore cause the amount of flux detected by the SPM to vary relative to that measured by MDI. A part of the difference between SPM and MDI is caused by differences in the behaviour of sunspot fields (see Sect. 3.3). A part may have to do with the different noise levels in the data.

\subsection{Threshold, identification of features and histograms}

Since for many purposes only magnetic fields above some threshold (which lies well above the noise level) are relevant, we have also carried out a comparison between the two instruments using only the pixels satisfying such a condition.

We now consider only pixels that harbour a significant magnetic field and compare facular fields, sunspot umbrae and penumbrae separately. Consequently we need to introduce a threshold for the magnetogram signal and to identify the various magnetic features. First, however, we rebin the SPM data to the same pixel size as the MDI data, i.e. from $1.14^{\prime \prime} \times 1.14^{\prime \prime}$ to $2^{\prime \prime} \times 2^{\prime \prime}$ pixels. This ensures that no bias due to the different pixel sizes enters the analysis. For the comparison we

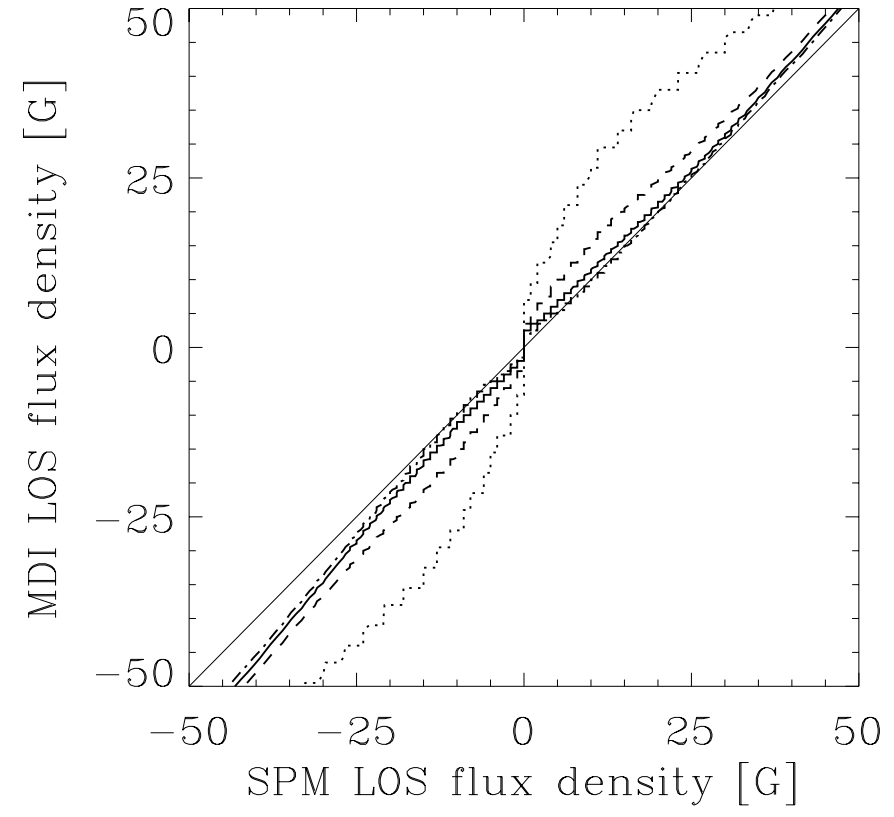

Fig. 6. Examples of histogram-equating curves for November 10, 2001, calculated with one SPM magnetogram and one standard 1-min (dotted line), one 5-min averaged (dashed line), one 20-min averaged (solid line) and one 56-min averaged MDI magnetogram (dash dotted line). The solid diagonal line represents the expectation values for identical SPM and MDI magnetograms.

consider 5-min integrated MDI magnetograms, since these are also the ones used for irradiance reconstructions. We are aware that the noise level of the 5-min integrated MDI magnetograms $(9 \mathrm{G})$ is higher than that of the SPM data. However, these are the lowest noise MDI magnetograms recorded daily.

Since the threshold must lie significantly above the noise and must be the same for both data sets (to allow a reliable comparison) its level is determined by the data set with the higher noise level, i.e. MDI. We choose:

$\left|B_{\mathrm{LOS}}(i, j)\right|>B_{\mathrm{LOS}}^{\mathrm{th}}(i, j)=3 \sigma_{\mathrm{mag}, \mathrm{MDI}}(i, j)$,

where $i$ and $j$ are indices identifying the pixels, $B_{\mathrm{LOS}}^{\text {th }}$ is the magnetic field threshold and $\sigma_{\text {mag, MDI }}$ is the noise level in the 5-min averaged MDI magnetograms, taken from Ortiz et al. (2002), cf. Sect. 2.2.

To identify sunspot umbrae and penumbrae we use continuum images. We follow Fligge et al. (2000b) and use a simple brightness threshold to distinguish between umbra, penumbra and the rest of the Sun. For MDI the brightness thresholds of umbrae and penumbrae used by Fligge et al. (2000b) are 0.60 and 0.90 , respectively. For SPM the use of the same thresholds leads to different umbral and penumbral areas, owing to the different wavelengths of the images and hence the different sunspot contrasts. Since it is important to subtract the same sunspot area from the SPM and MDI magnetograms, we determined the thresholds for the SPM continuum images by requiring the average sunspot umbral and penumbral areas to be the same for both data sets. This resulted in brightness threshold contrasts for SPM of 0.64 and 0.92. Examples of masks with identified umbrae and penumbrae are plotted in Fig. 7. MDI and SPM data yield nearly identical masks. 


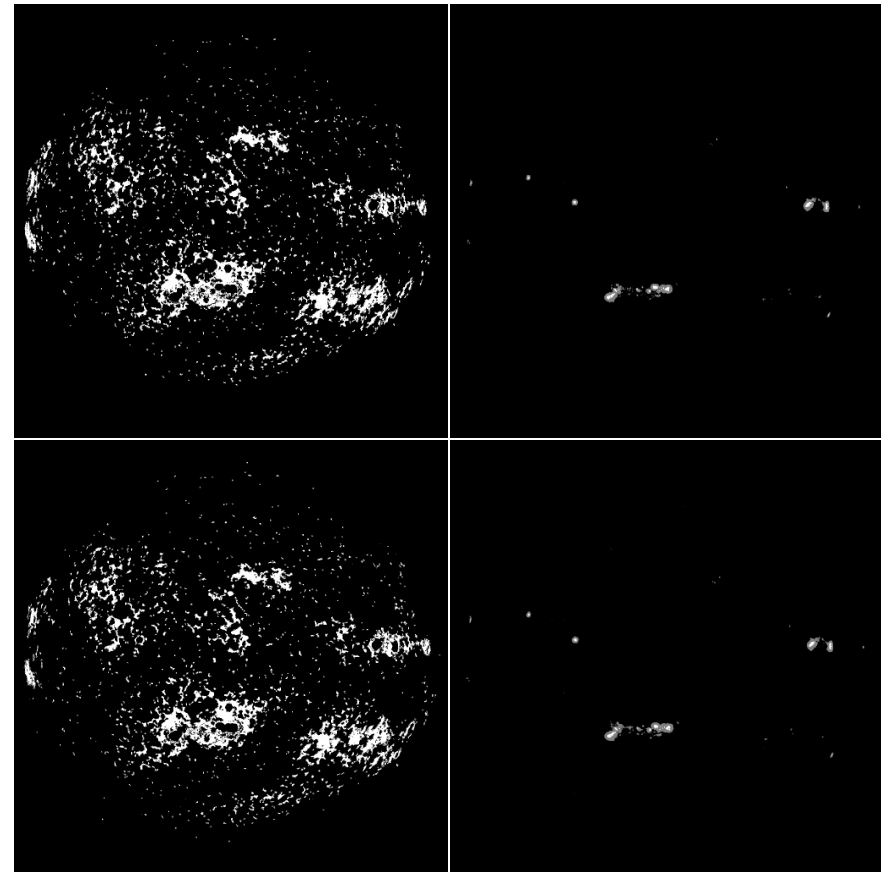

Fig. 7. Examples of facular (left) and spot (right) masks for November 10, 2001. The upper frames represent masks obtained from SPM data, the lower frames from MDI data. In the spot masks the umbral area is plotted white, while the penumbral area is grey.

Next we determine the facular distribution. For this purpose we start from magnetogram masks in which umbral and penumbral areas, as obtained from continuum images, have been removed. Faculae are then identified according to Eq. (1) as all locations where $\left|B_{\mathrm{LOS}}\right| \geq B_{\mathrm{LOS}}^{\text {th }}$. Figure 7 gives an example of facular masks based on MDI and SPM magnetograms.

A visual inspection of the masks shows that the MDI and SPM data coincide well. To compare the two data sets in more detail we create histograms of the umbral, penumbral and facular distributions, which will be discussed in Sect. 3.3. For umbrae and penumbrae we study histograms $N_{\mathrm{u}}\left(\mu, B_{\mathrm{LOS}}\right)$ and $N_{\mathrm{p}}\left(\mu, B_{\mathrm{LOS}}\right)$, respectively, while we consider the distribution $N_{\mathrm{f}}(\mu, B)$ for faculae, where $B=\left|B_{\mathrm{LOS}}\right| / \mu$. The factor $1 / \mu$ in $B$ takes into account that a magnetogram underestimates the true flux in pixels close to the limb since facular and network fields on the Sun are mainly vertical (Martínez Pillet et al. 1997; Bernasconi 1997). The $\mu$ - and $B$-values ( $B_{\mathrm{LOS}}$ in the case of spots) have been binned linearly, with $\Delta \mu=0.01$ and $\Delta B=5 \mathrm{G}$ (for spots $\Delta B_{\mathrm{LOS}}=5 \mathrm{G}$ ), respectively, resulting in a grid with $101 \times 241$ bins. Entries in the histograms with $\mu \leq \mu_{\text {th }}=0.1$ are set to zero, in order to avoid artifacts introduced by the presence of possible data gaps at the very limb.

\subsection{Comparisons of the facular, umbral and penumbral fields as well as sunspot areas}

In the following, we compare histograms of the umbral, $N_{\mathrm{u}}\left(\mu, B_{\mathrm{LOS}} ; t\right)$, penumbral, $N_{\mathrm{p}}\left(\mu, B_{\mathrm{LOS}} ; t\right)$ and facular distributions, $N_{\mathrm{f}}(\mu, B ; t)$, obtained from the MDI and SPM data as described in Sect. 3.2. The distributions are studied as a function of $\mu$ or as a function of the magnetic flux, either for a single day (characterised by the variable $t$ ) or for the average over the selected 24 days (in which case we omit the time variable $t$ ).

Figure 8 shows the MDI (dashed line) and SPM (solid line) histograms of the umbral and penumbral area vs. $\mu, N_{\mathrm{u}}(\mu ; t)$ and $N_{\mathrm{p}}(\mu ; t)$, deduced from continuum images recorded on November 10, 2001. These histograms have been integrated over $B_{\mathrm{LOS}}$. It is evident that the MDI and SPM histograms agree very well with each other. This good agreement implies that for the chosen thresholds not only are the average areas the same, but also the location and distribution on the solar surface. Effects due to sunspot evolution or interpolation between different pixel sizes do not appear to play a significant role.

It is instructive to compare also the magnetic fields in spots obtained from MDI and SPM. Figure 9 shows the umbral and penumbral field distributions integrated over $\mu$ and averaged over the selected 24 days. As expected from the histogramequating curves (cf. Fig. 5) the MDI magnetic fields are shifted towards greater values compared to SPM data. The magnetic flux in SPM magnetograms integrated over the whole solar disk is $16.7 \%$ smaller in umbral areas and $24.7 \%$ smaller in penumbral areas than in MDI magnetograms. These values partly reflect the slopes of the histogram-equating curves given in Table 1. The umbral fields returned by both instruments are clearly too low, with the number of pixels peaking at around $1000 \mathrm{G}$ or less, whereas we would expect a value higher by a factor of 1.5-2. For example Keppens \& Martínez Pillet (1996), using Advanced Stokes Polarimeter data, find that in the umbrae of sunspots $B_{\mathrm{Z}}$ lies between $1000 \mathrm{G}$ and $2500 \mathrm{G}$ (cf. Solanki 2003). Although we expect somewhat lower values, due to the inclusion of sunspots near the limb in our sample, this alone cannot explain the low $B_{\mathrm{LOS}}$ values returned by MDI and in particular SPM. Possible problems with the determination of umbral fields with MDI have been pointed out earlier (e.g. Solanki \& Rüedi 2003; R. Bush \& N. Meunier, private communication). Stray light is one possible culprit for reduced magnetic flux values in sunspot umbrae. If this is the main cause of the low measured values, then our analysis suggests that stray light effects are larger in the SPM than in MDI. Note that the different magnetic field values from MDI and SPM have no influence for the reconstruction of the total solar irradiance because there we use the umbral and penumbral areas, which differ by only $3.4 \%$ and $0.2 \%$, respectively.

The MDI (dashed line) and SPM (solid line) histograms for 2001 November 10 of the facular distributions integrated over $\mu$ are plotted vs. $B_{\mathrm{LOS}} / \mu$ in Fig. $10 \mathrm{a}$ and integrated over $B_{\mathrm{LOS}} / \mu$ vs. $\mu$ in Fig. $10 \mathrm{~b}$. The MDI and the SPM facular histograms have small differences. At small $B_{\mathrm{LOS}} / \mu$ values more SPM pixels show a signal, while more MDI pixels show a signal with $B_{\mathrm{LOS}} / \mu \gtrsim 300 \mathrm{G}$. When plotted vs. $\mu$ more SPM pixels lie above the threshold at almost all $\mu$. To check to what extent these differences are exemplary for the whole time series, we compare the histograms as follows. For each of the 24 selected days distributed in time between 1996 and 2001 we compute the MDI and SPM histograms of the facular distributions after integration over $\mu$ and, alternatively, after integration over $B_{\mathrm{LOS}} / \mu$. Then we form the averages over the 

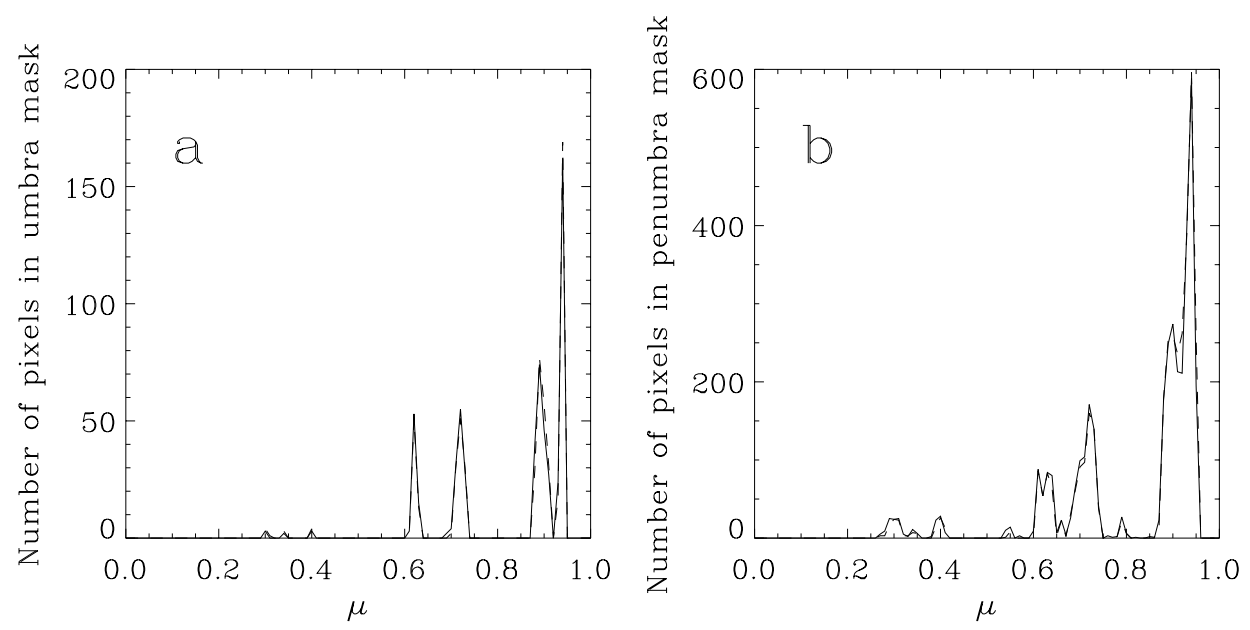

Fig. 8. Histogram of the umbral a) and penumbral b) mask vs. $\mu$ for November 10, 2001. Solid lines represent SPM data, dashed lines MDI data. $\mu=1$ is the disk centre; $\mu=0$ is the limb.
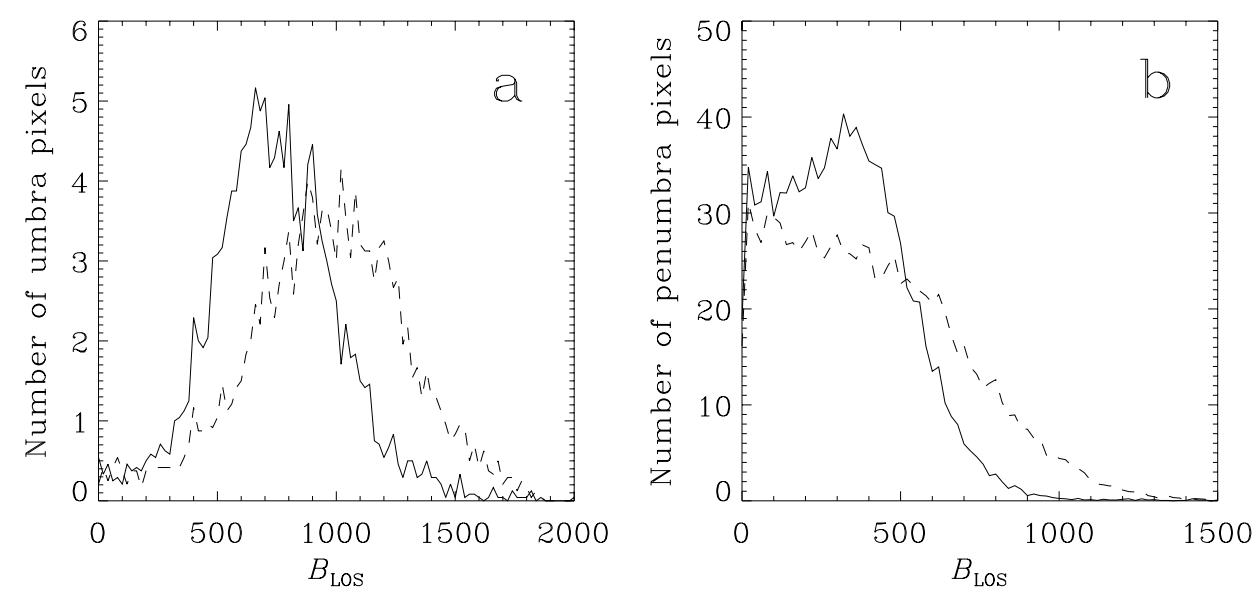

Fig. 9. 24-day averaged histograms of the umbral and penumbral magnetic distributions. a) The number of umbral points vs. $B_{\mathrm{L} O S}$. b) The number of penumbral points vs. $B_{\text {LOS. }}$ Solid lines represent SPM data, dashed lines MDI data.

24 selected days of both expressions, $N_{\mathrm{f}}^{i}(B)$ and $N_{\mathrm{f}}^{i}(\mu)$, respectively, where $i$ stands for MDI or SPM.

These 24-day averaged histograms are illustrated in Fig. 11. $N_{\mathrm{f}}^{\mathrm{SPM}}(B)$ and $N_{\mathrm{f}}^{\mathrm{SPM}}(\mu)$ have about $9.2 \%$ more pixels than $N_{\mathrm{f}}^{\mathrm{MDI}}(B)$ and $N_{\mathrm{f}}^{\mathrm{MDI}}(\mu)$. The histograms plotted in Fig. 11 are rather similar to those in Fig. 10, except that the scatter is now considerably lower. Above the threshold both the instruments exhibit a rapid drop of the number of pixels with $B_{\mathrm{LOS}} / \mu$. This drop cannot be well described by a power law and is less rapid than an exponential. This agrees with earlier such histograms (e.g. Harvey \& White 1999). Note that the shape of the distribution seen in Fig. 11b does not say anything about the visibility of the magnetic features from the disk centre to the limb, since the surface area covered by individual $\mu$ bins strongly depends on $\mu$ itself. In addition, the activity belts affect the shape of the distribution in a non-trivial way.

To highlight the difference between the 24-day averaged MDI and SPM histograms $N_{\mathrm{f}}^{i}(B)$ and $N_{\mathrm{f}}^{i}(\mu)$, we plot in Fig. 12 two relative differences $d_{\mathrm{f}}^{\text {rel }}(B)$ and $d_{\mathrm{f}}^{\text {rel }}(\mu)$ determined as

$d_{\mathrm{f}}^{\text {rel }}(B)=2 \cdot \frac{N_{\mathrm{f}}^{\mathrm{SPM}}(B)-N_{\mathrm{f}}^{\mathrm{MDI}}(B)}{N_{\mathrm{f}}^{\mathrm{SPM}}(B)+N_{\mathrm{f}}^{\mathrm{MDI}}(B)}$ and

$d_{\mathrm{f}}^{\mathrm{rel}}(\mu)=2 \cdot \frac{N_{\mathrm{f}}^{\mathrm{SPM}}(\mu)-N_{\mathrm{f}}^{\mathrm{MDI}}(\mu)}{N_{\mathrm{f}}^{\mathrm{SPM}}(\mu)+N_{\mathrm{f}}^{\mathrm{MDI}}(\mu)}$.

Figure 12 reveals the complex relationship between SPM and MDI magnetograms even more clearly than before. The number of pixels exhibiting a signal with a given $B_{\mathrm{LOS}} / \mu$ value can differ by up to a factor of 2 (for $B_{\mathrm{LOS}} / \mu \approx 400-600 \mathrm{G}$ ). The SPM data exhibit more magnetic flux at small and large $B_{\mathrm{LOS}} / \mu$ values, while MDI dominates at intermediate values. SPM shows more facular pixels at all $\mu$ values, but mainly at large and small $\mu$.

In the following we briefly discuss the relation between the histogram-equating curves (Sect. 3.1) and the facular histograms considered in this section. From the histogramequating curves comparing 5-min MDI and SPM magnetograms we have obtained a slope of 1.268 (see Table 1). Therefore we would expect to get more facular pixels in MDI magnetograms. However, in the histogram-equating curves we have considered all magnetogram pixels, whereas to obtain the facular histograms several filters have been applied. First, the sunspot areas have been removed, then the 

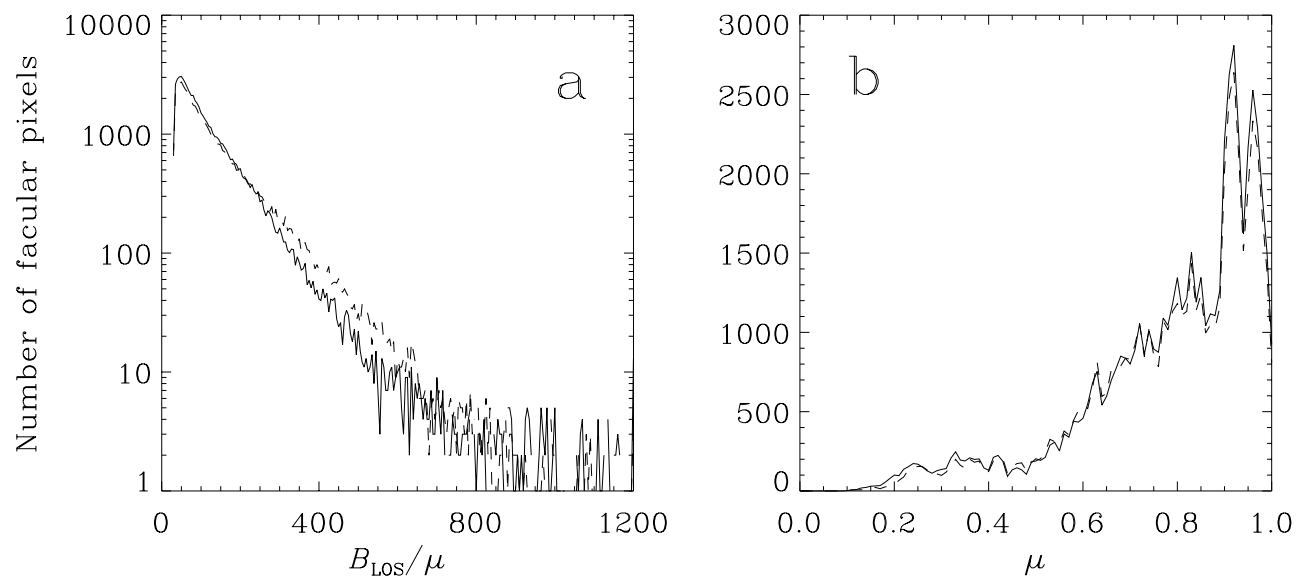

Fig. 10. Histograms of the facular distributions for November 10, 2001. a) The number of facular points vs. $B_{\mathrm{LOS}} / \mu$; b) the same quantity vs. $\mu$. Solid lines represent SPM data, dashed lines MDI data.
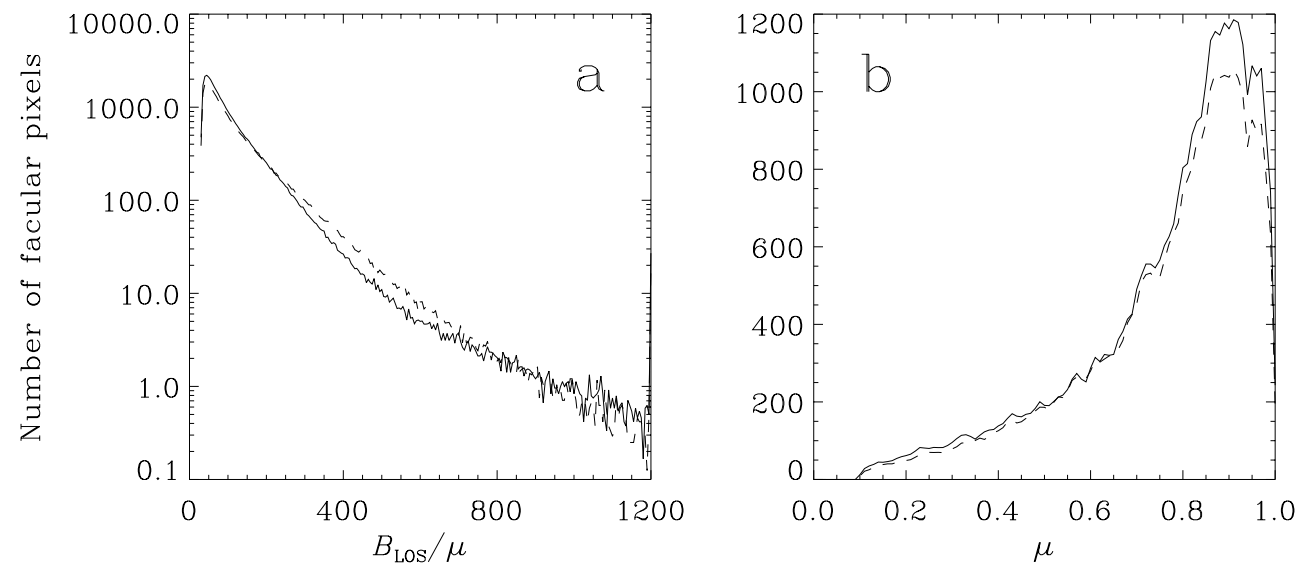

Fig. 11. The same as Fig. 10, but now for 24-day averaged histograms of the facular distributions.
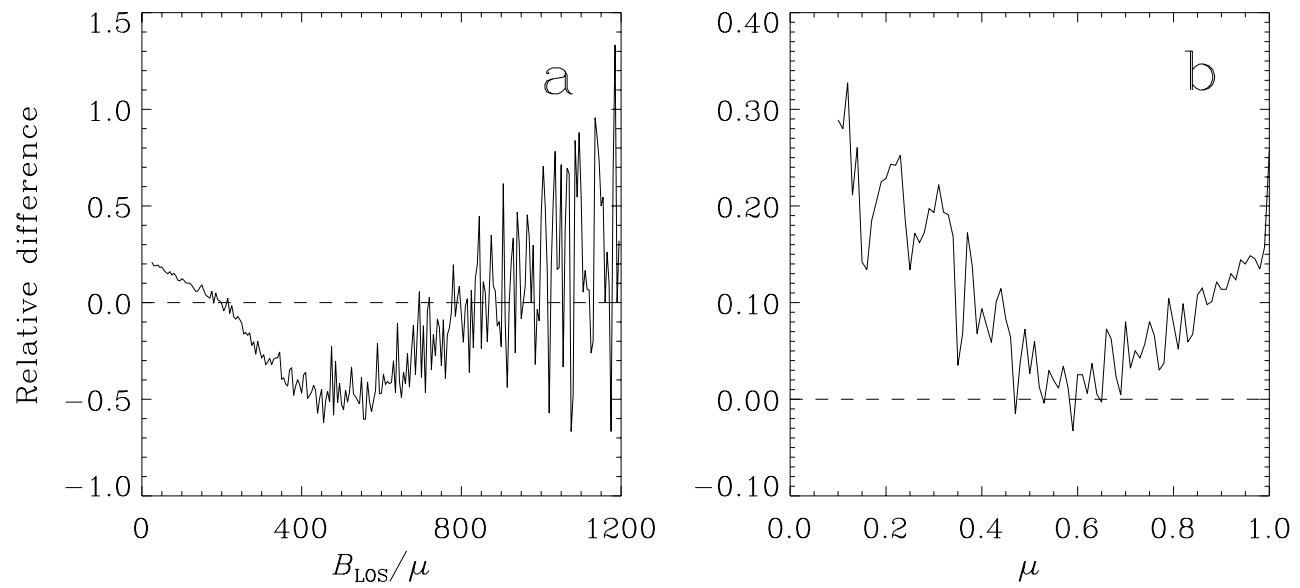

Fig. 12. Relative difference of the facular magnetic distributions plotted vs. $B_{\mathrm{LOS}} / \mu \mathbf{a}$ ) and vs. $\mu$ b). Positive values indicate that SPM data show more pixels with signal at that $B_{\mathrm{LOS}} / \mu$ or $\mu$ value. The solid lines represent relative differences based on histograms, averaged over 24 individual days between 1997 and 2001. Dashed lines indicate $d_{\mathrm{f}}^{\text {rel }}=0$.

magnetic threshold has been applied, and finally a further filter has been used to reduce noise effects. Note that we employ the same magnetic threshold, and the spot areas in both MDI and SPM data coincide and thus do not influence the differences in the facular histograms. The last filter removes isolated magnetic pixels which are considered as unphysical (see Fligge et al. 2000b). Since the noise is considerably larger in MDI than in SPM magnetograms this final filter removes many more MDI pixels. As a result, the number of SPM facular pixels even exceeds the corresponding number of MDI pixels (Fig. 12b). These additional SPM pixels are shifted towards smaller $B_{\mathrm{LOS}} / \mu$ values (Fig. 12a) because on average an 


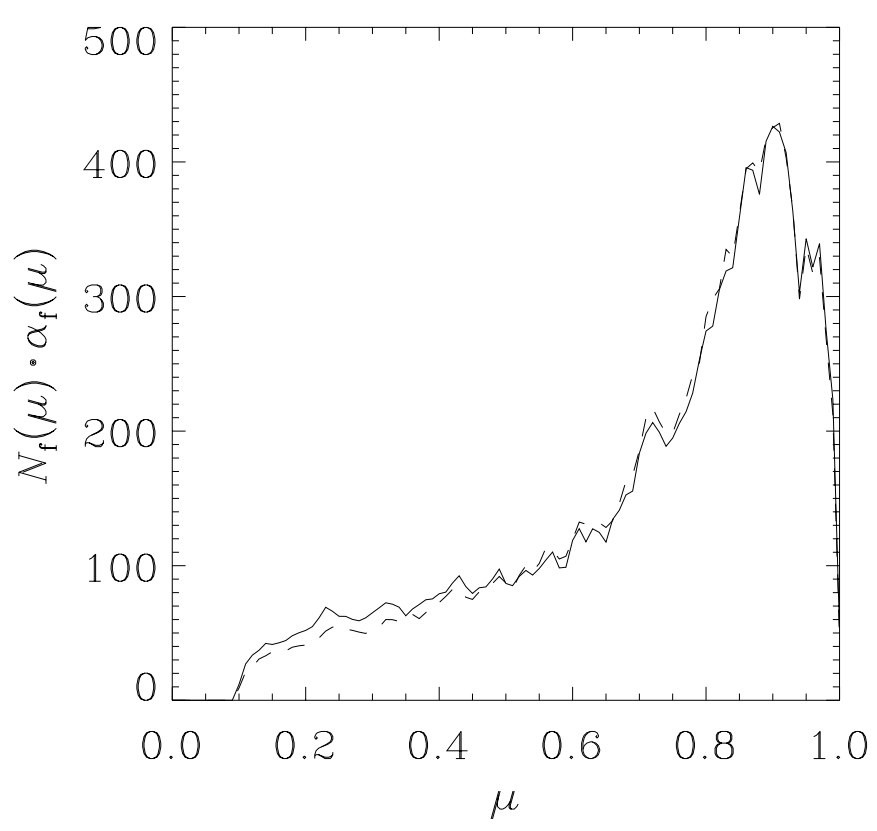

Fig. 13. Facular area as a function of $\mu$ using 24-day averaged histograms for SPM data (solid line) and MDI data (dashed line). The variable $\alpha_{\mathrm{f}}$, defined in Eq. (6), is the facular filling factor per pixel, while $N_{\mathrm{f}}$ is the number of facular pixels.

SPM pixel has a smaller magnetic signal than an MDI pixel, as follows from the histogram-equating curves.

Interestingly, the difference in total facular magnetic flux above the threshold is almost negligible. The relative difference of the number of facular SPM and MDI pixels integrated over all magnetic field strengths and $\mu$ values is

$\Delta N_{\mathrm{f}}^{\mathrm{rel}}=2 \cdot \frac{\sum_{B}\left[N_{\mathrm{f}}^{\mathrm{SPM}}(B)-N_{\mathrm{f}}^{\mathrm{MDI}}(B)\right]}{\sum_{B}\left[N_{\mathrm{f}}^{\mathrm{SPM}}(B)+N_{\mathrm{f}}^{\mathrm{MDI}}(B)\right]}=0.092$,

i.e. there are $9.2 \%$ more facular SPM pixels. However, the relative flux difference

$\Delta \phi_{\mathrm{f}}^{\mathrm{rel}}=2 \cdot \frac{\sum_{B} B \cdot\left[N_{\mathrm{f}}^{\mathrm{SPM}}(B)-N_{\mathrm{f}}^{\mathrm{MDI}}(B)\right]}{\sum_{B} B \cdot\left[N_{\mathrm{f}}^{\mathrm{SPM}}(B)+N_{\mathrm{f}}^{\mathrm{MDI}}(B)\right]}=-0.012$,

so that the MDI flux actually exceeds the SPM flux, although by a very small amount. There are more SPM facular pixels but this is compensated by the smaller average magnetic flux per pixel.

In the irradiance reconstructions we use a facular filling factor, $\alpha_{\mathrm{f}}$, which scales linearly with $B_{\mathrm{LOS}} / \mu$ up to a fixed saturation value (see Sect. 4 and Eq. (6) for details). Since this filling factor is related to the magnetic flux, it is more relevant to compare MDI and SPM fluxes than differences in the number of facular pixels. Because the magnetic flux agrees well it is not surprising that the irradiance reconstructions give very similar results whether employing MDI or SPM data, as will be discussed in the following two sections. If we consider in Eq. (5) also the saturation value inherent in the definition of $\alpha_{\mathrm{f}}$ we obtain a relative difference of 0.008 , which is smaller still and negligible as far as the irradiance reconstructions are concerned. Figure 13 compares the facular areas in the MDI and
SPM data as used in the reconstructions of the total solar irradiance. The two curves are very similar for all $\mu$ values, in contrast to the big differences in the number of facular pixels seen in Fig. $11 \mathrm{~b}$ for $\mu \gtrsim 0.8$.

\section{Total solar irradiance reconstructions: The model}

For reconstructing variations of total solar irradiance we employ the 4-component model of Fligge et al. (2000a,b) and Krivova et al. (2003). The basic assumption of this model is that all irradiance changes are caused by the evolving distribution of the magnetic field on the solar surface. We divide the solar photosphere into the following four components: quiet Sun (subscript q in the subsequent discussion), sunspot umbrae (u), sunspot penumbrae (p) and faculae (f). In order to describe the variation of solar irradiance with time, $t$, two input ingredients are required: the intensity of each photospheric component and the fraction of the solar surface covered by this component.

Intensities, $I(\mu, \lambda)_{\mathrm{q}, \mathrm{u}, \mathrm{p}, \mathrm{f}}$, do not change with time but depend on the wavelength $\lambda$ and the heliocentric angle, $\theta$ ( $\mu=$ $\cos \theta$ ). Unruh et al. (1999) calculated $I(\mu, \lambda)_{\mathrm{q}, \mathrm{u}, \mathrm{p}, \mathrm{f}}$ employing the ATLAS9 code of Kurucz (1992) from the following planeparallel model atmospheres. The standard model atmosphere FAL-C (Fontenla et al. 1993) is utilized for the quiet Sun. Umbrae and penumbrae are described by the appropriate radiative equilibrium models (Kurucz 1991; cf. Severino et al. 1994; Solanki 1997) having effective temperatures $T_{\text {eff }}=4500 \mathrm{~K}$ and $T_{\text {eff }}=5400 \mathrm{~K}$, respectively. A slightly modified version of FAL-P (Fontenla et al. 1993; see also Unruh et al. 1999) is chosen for the faculae.

The fraction of the solar surface occupied by each model component is a function of time and it is this temporal evolution which is responsible for variations of the solar total (and spectral) irradiance. To find the distribution of the magnetic field on the Sun's surface at a given instant of time we use daily sequences of SPM or MDI full-disk magnetograms and intensity images described in Sect. 2. To be able to compare the SPM reconstructions of TSI directly with those from MDI, we use SPM images (magnetogram and associated continuum image) with pixel size increased to that of MDI. In addition we use the same time-independent threshold for the lowest flux per pixel as for the MDI data, i.e. $3 \sigma_{\mathrm{mag}, \text { MDI }}$. To decrease the magnetic noise level we use averages over 5 single MDI magnetograms. Since the SPM noise level is considerably lower, employing the relatively high threshold dictated by noise in MDI magnetograms should not adversely affect the TSI reconstruction using SPM data.

For the reconstruction we use the histograms produced in Sect. 3.2. Previously masks such as those shown in Fig. 7 had been used (e.g. Fligge et al. 2000a,b). The advantages of using the histograms rather than the masks are of a practical nature. The contribution to the irradiance from any given pixel depends only on whether it is an umbral, penumbral or facular pixel, on the $\mu$-value of the pixel and, for facular pixels, on the filling factor $\alpha_{\mathrm{f}}$ defined in Eq. (6). Therefore the masks are a very inefficient way of storing the information needed for irradiance reconstructions. The histograms require 

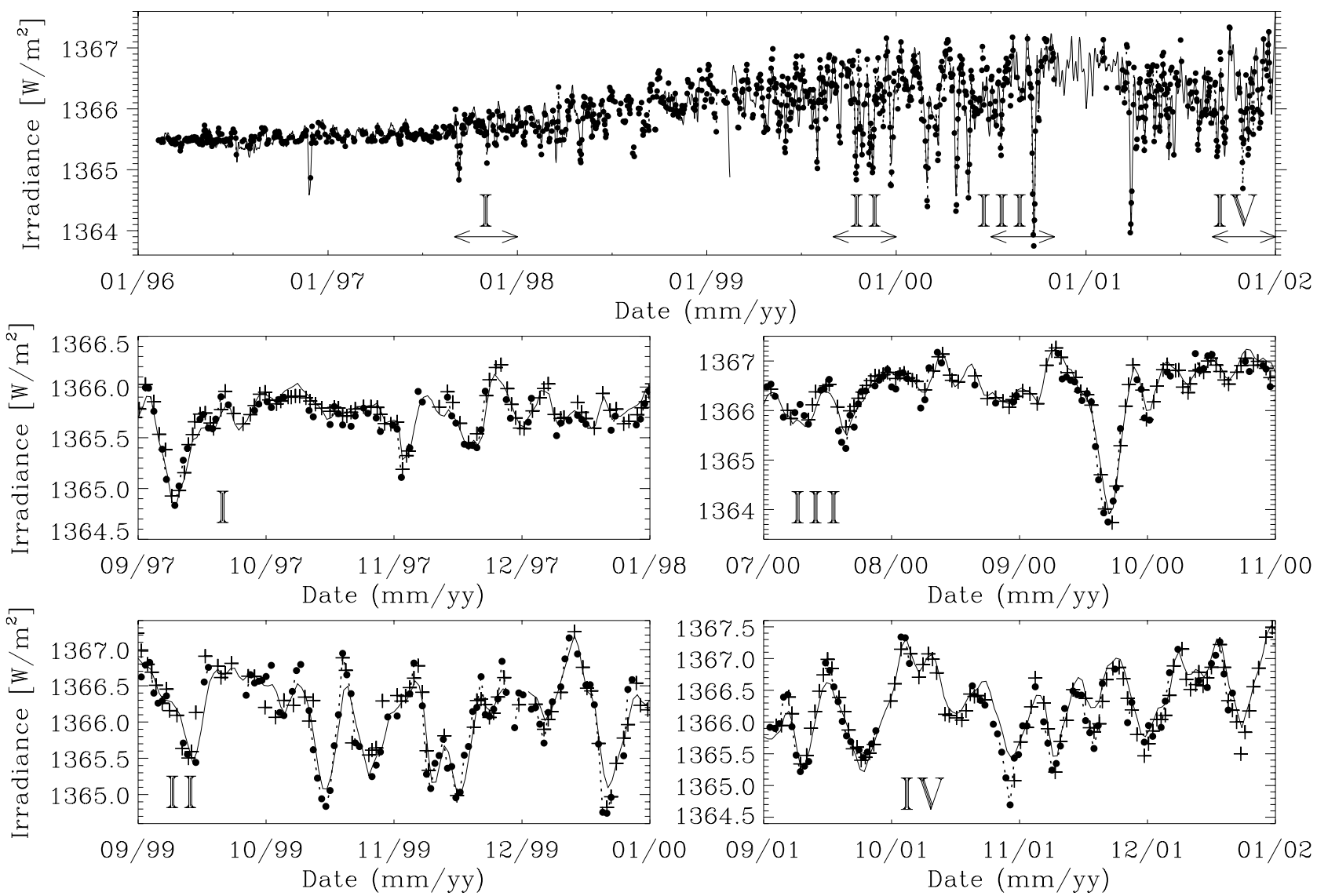

Fig. 14. Total solar irradiance measured by VIRGO (solid line) and reconstructed based on SPM data (filled circles, connected by the dotted curve when there are no data gaps) for 1161 individual days between 1996 and 2001, i.e. from the minimum of cycle 23 to its maximum (top panel). The middle and lower panels show enlargements of four shorter intervals at different activity levels. The times corresponding to these zoom-ins are marked in the top panel by roman numerals. A reconstruction based on MDI data is represented (middle and lower panels only) by the plus signs.

roughly 130 times less disk space and result in irradiance reconstructions that are much faster to compute. Reconstructions using MDI, but based on histograms, gave identical results to those employing the masks.

Sunspots represent the largest concentrations of magnetic flux on the solar surface and are much bigger than the size of an individual pixel. Therefore, a pixel falling within a sunspot is considered to be entirely "filled" by it, i.e. the filling factor $\alpha_{\mathrm{u}, \mathrm{p}}(i, j)=1$. For faculae, however, this is not the case. Therefore, their filling factor $\alpha_{\mathrm{f}}(i, j, B)$ is taken to depend linearly on the magnetogram signal $B_{\mathrm{LOS}}(i, j)$ :

$\alpha_{\mathrm{f}}(i, j, B)=\min \left\{\frac{\left|B_{\mathrm{LOS}}(i, j)\right| / \mu(i, j)}{B_{\mathrm{sat}}} ; 1\right\}$

The single free parameter of the model $B_{\text {sat }}$ signifies the average field strength at which the brightness of magnetic elements saturates (e.g. Solanki \& Stenflo 1984; Foukal \& Fowler 1984; Ortiz et al. 2002; see Fligge et al. 2000b, for the details). In the following we keep $B_{\text {sat }}=280 \mathrm{G}$ fixed at the value found by Krivova et al. (2003) from a comparison of an MDI-based reconstruction with VIRGO measurements.
Finally, the solar irradiance is calculated by summing over contributions from each component:

$$
\begin{aligned}
S(\lambda, t)= & \sum_{\mu}\left[N_{\mathrm{u}}(\mu ; t) I_{\mathrm{u}}(\mu ; \lambda)+N_{\mathrm{p}}(\mu ; t) I_{\mathrm{p}}(\mu ; \lambda)\right. \\
& +N_{\mathrm{f}}(\mu, B ; t) \alpha_{\mathrm{f}}(\mu, B) I_{\mathrm{f}}(\mu ; \lambda) \\
& +\left(1-N_{\mathrm{u}}(\mu ; t)-N_{\mathrm{p}}(\mu ; t)\right. \\
& \left.\left.-N_{\mathrm{f}}(\mu, B ; t) \alpha_{\mathrm{f}}(\mu, B)\right) I_{q}(\mu ; \lambda)\right]
\end{aligned}
$$

where $\alpha_{\mathrm{f}}(i, j, B)$ are found using Eq. (6) and then summed over pixels with the same $\mu$ to give $\alpha_{\mathrm{f}}(\mu, B)$.

\section{Total solar irradiance reconstructions: Results for cycle 23}

\subsection{Results for cycle 23}

In this section we consider how SPM data compare with MDI data for modelling solar irradiance. The model described in Sect. 4 has been used to reconstruct the total solar irradiance for 1161 days between February 1996 and December 2001 based on SPM data. The results are presented in Fig. 14 (filled circles connected by dotted curve). The VIRGO total irradiance measurements (level 2; Fröhlich \& Finsterle 2001) are given by the solid lines. The top panel demonstrates that the increase of 


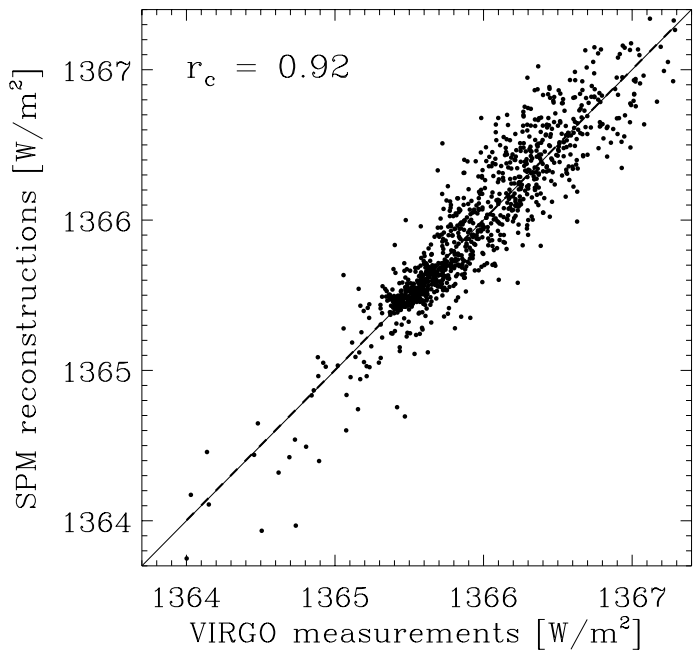

Fig. 15. Modelled total solar irradiance based on SPM data vs. VIRGO measurements. The correlation coefficient, $r_{\mathrm{c}}$, is indicated. The solid diagonal line represents the expectation values for a perfect model fit, a regression (dashed line) is hardly distinguishable.

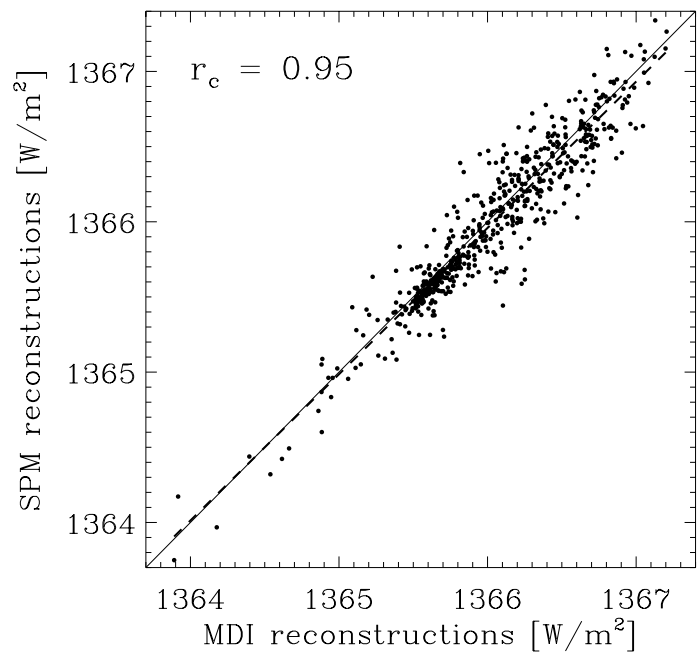

Fig. 16. The same as Fig. 15, but now plotted vs. MDI-based reconstructions of TSI.

total solar irradiance from the minimum of solar cycle 23 to its maximum is very well reproduced. The two middle and the two lower panels display extracts on an enlarged scale at different phases of the solar cycle. The plus signs are the MDI reconstruction by Krivova et al. (2003). These four panels reveal that the short-term variations of the total solar irradiance are also very well reproduced. By eye it is difficult to tell which reconstruction reproduces the data better. In Fig. 15 the reconstructed value based on SPM data is plotted against the measured irradiance (VIRGO measurements). The resulting correlation coefficient is $r_{\mathrm{c}}=0.92$. This is slightly lower than the value of 0.96 obtained by Krivova et al. (2003) between MDI based reconstructions and VIRGO measurements. The $r_{\mathrm{c}}$ obtained by the SPM reconstruction must be considered to be a lower limit, since the parameter $B_{\text {sat }}$ used here is optimised for MDI. The choice of an appropriate $B_{\text {sat }}$ could improve the SPM-based fit further. Note also that there is practically no bias between high and low irradiance values. The linear regression to the dots (dashed line) is practically indistinguishable from the expectation value (solid line). In Fig. 16 the reconstructed value based on SPM data is plotted against the reconstructed value based on MDI data. The correlation coefficient $r_{\mathrm{c}}=0.95$ is basically the same as the correlation between the reconstruction using MDI and VIRGO measurements.

\subsection{The SoHO data gaps in 1998 and early 1999}

Since MDI, like VIRGO, is an instrument on board SoHO, it cannot help to bridge the data gap in VIRGO during the loss of contact with SoHO in 1998 and 1999. However, SPM data do not suffer from this and can be used to compute the TSI in the gap. These values can be compared with the composite of data from VIRGO, ACRIM II and other radiometers, adjusted to the Space Absolute Radiometric Reference (Crommelynck et al. 1995), constructed by Fröhlich (2003). In the composite TSI record ACRIM II data are used to fill in the SoHO data gap in 1998 and early 1999. Figure 17 shows the reconstructed TSI values based on SPM data as well as the composite of measured irradiance over an interval that contains the SoHO data gaps in 1998 and early 1999. Since VIRGO is offset by $0.056 \mathrm{Wm}^{-2}$ relative to the composite, the latter has been shifted upwards by this amount (note that the reconstructions only compute the irradiance change, not its absolute value). To distinguish between VIRGO and ACRIM II, the data from the latter instrument is indicated by a thicker line. Figure 17 shows a relatively good correspondence between the reconstructions and all the data, indicating that any offsets between VIRGO and ACRIM II have been correctly compensated in the composite (at least for the rather limited interval of time considered here).

\subsection{Results in 1996}

During the considered interval (1996-2001) there is one period, basically the second half of 1996, which strikes the eye because of a mismatch between data and model, both MDI and SPM-based (see Fig. 18). Although the maximum difference, about $0.2 \mathrm{Wm}^{-2}$, is not particularly large, as can be seen by considering Fig. 15, this period is striking because the deviation persists so consistently for such a length of time. As long as only a reconstruction based on MDI data was available, there were three possible explanations for the discrepancy. 1) Errors in the MDI data; 2) non-validity of the basic assumption of the model (irradiance variations are caused exclusively by surface magnetism); 3) problems with the calibration of VIRGO in this period. Both 1) and 3) are possibilities since SoHO had not been flying very long at that time and degradation of sensitivity may have been higher than expected.

From Fig. 18 it can be deduced that the reconstructions based on SPM lie on average halfway between those based on MDI and the VIRGO data. This suggests that explanation 1) is not likely to explain the whole discrepancy, but may be the cause of part of it. 


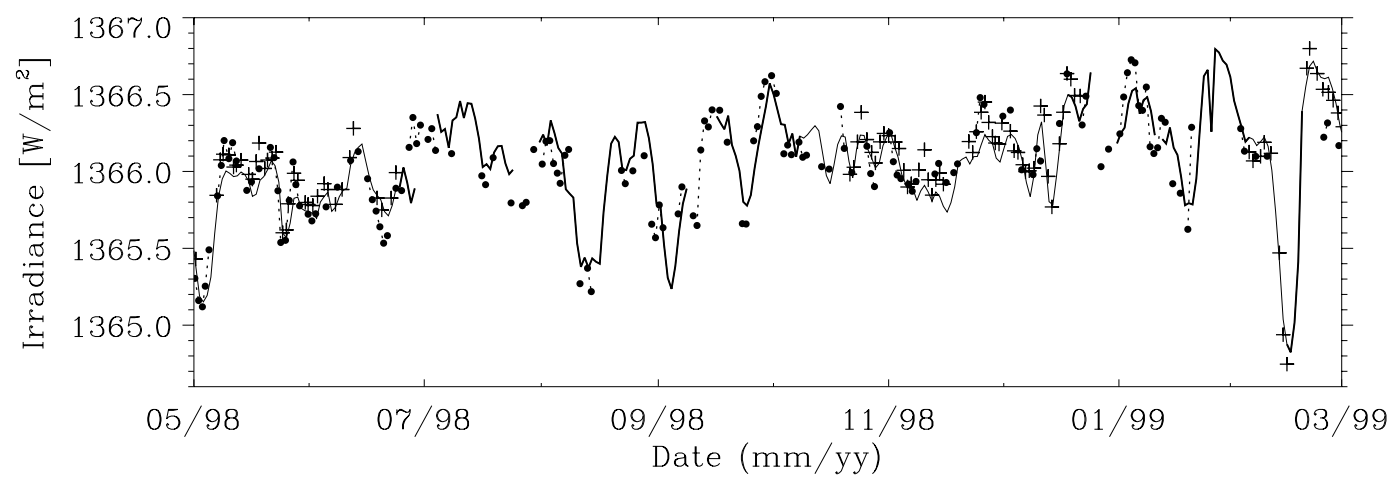

Fig. 17. Total solar irradiance measured by VIRGO (thin solid line) and by ACRIM II (thick solid line) and reconstructed based on SPM data (filled circles connected by dotted curve when there are no data gaps) during the SoHO failure in 1998 and early 1999. The ACRIM II data, adjusted to the Space Absolute Radiometric Reference data, are part of a composite record, constructed by Fröhlich (2003) (see text for details). A reconstruction based on MDI data is represented by the plus signs.

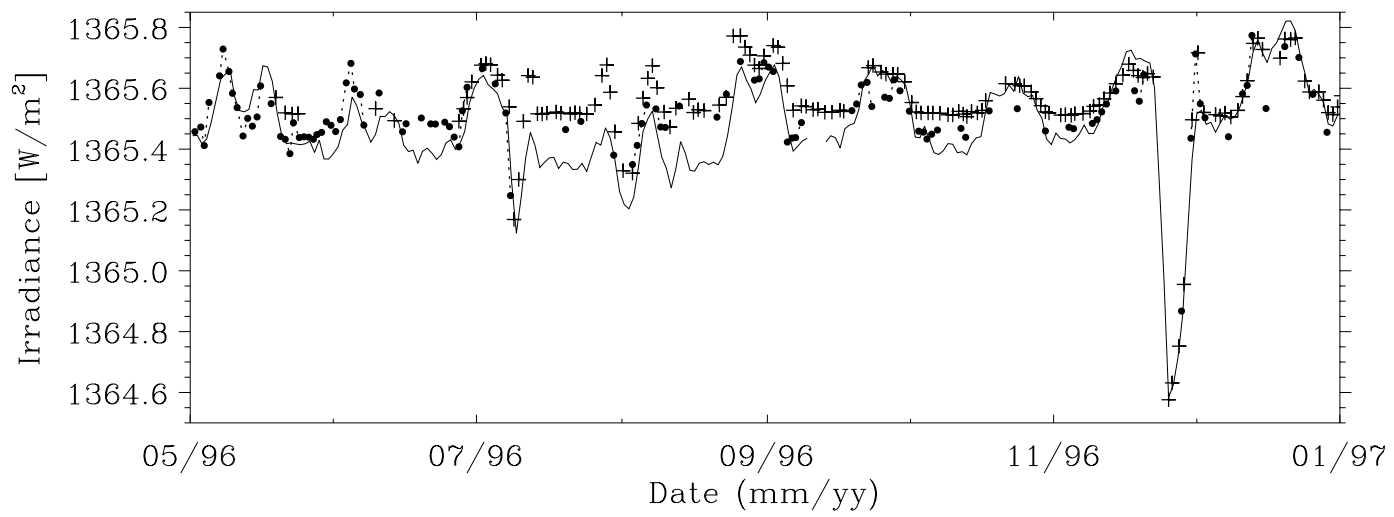

Fig. 18. Total solar irradiance measured by VIRGO (solid line) and reconstructed based on SPM data (filled circles connected by dotted curve when there are no data gaps) and on MDI data (plus signs) for one period of eight months in 1996.

\section{Conclusions}

The main aim of this paper is to compare two of the most widely used sets of magnetograms, namely those recorded by MDI on SoHO and by the Vacuum Tower Spectropolarimeter (SPM) on Kitt Peak. To this end we have applied different techniques. The results are, on the one hand, heartening in that, globally, the two sets of magnetograms appear to give the same solar magnetic flux to within roughly $1 \%$. This difference in magnetic flux is remarkably small if we keep in mind that the measurements are made with instruments of different type, that the field strengths are extracted using different techniques and that spectral lines with different Zeeman and temperature sensitivities are used.

Frustratingly, however, we do not find a simple number or relation to convert MDI magnetograms into SPM ones or vice versa. They exhibit a complex relationship (see Fig. 12), which not only depends on the magnetogram signal and $\mu$ value, but also on the technique used to compare them, the threshold used etc. However, the two instruments give almost identical values for the total magnetic flux in facular regions if the same threshold is applied. Both seem to underestimate the magnetic flux in sunspot umbrae, however, and give different values.

We have also compared the continuum images recorded by the two instruments and have found these to give almost identical locations and areas of sunspot umbrae and penumbrae if the appropriate brightness thresholds are chosen.

Finally, we have tested how well the SPM data can be used to reconstruct total solar irradiance variations. We find that the reconstruction agrees rather well with the VIRGO data and with the MDI reconstruction, even if the free parameter is forced to have exactly the same value as the one employed by Krivova et al. (2003) for the MDI reconstruction. This result also gives us the possibility to calculate the reconstructions of TSI based on the NASA/NSO Spectromagnetograph at the NSO/KPVT, for the whole period of time that these data are available, i.e. from 1992 to 2001. These reconstructions and their comparison with irradiance measurements will be the subject of a separate publication.

We consider in detail the periods during which no MDI and VIRGO data were available in 1998 and 1999, as well as 5-6 months in 1996 in which the MDI reconstruction shows a systematic offset relative to VIRGO data. In the latter case SPM reconstructions lie between those made with MDI and the VIRGO data. This suggest that a part of the offset may be due to MDI systematics, but the rest could either be caused by a process independent of surface magnetism that produced irradiance variations during that interval or be due to a small problem with VIRGO calibrations. 
Acknowledgements. We thank H. P. Jones for providing information on the SPM and the histogram-equating technique, and M. Lockwood for helpful comments on the manuscript. M. Fligge and C. Frutiger provided useful advice at the beginning of this project, which we gratefully acknowledge. The work of T. Wenzler was carried out under Poly project "Variability of the Sun and Global Climate" at ETH Zurich. The excellent working conditions and the generous support are herewith gratefully acknowledged.

\section{References}

Bernasconi, P. N. 1997, Stokes Vector-Polarimetry: Observation and Analysis of Solar Magnetic Fields, Ph.D. Thesis, ETH, Zurich

Chapman, G. A., Cookson, A. M., \& Dobias, J. J. 1996, J. Geophys. Res., 101, 13541

Crommelynck, D., Fichot, A., Lee, R. B., \& Romero, J. 1995, Adv. Space Res., 16, 17

Fligge, M., Solanki, S. K., Meunier, N., \& Unruh, Y. C. 2000a, ESA, SP-463, 117

Fligge, M., Solanki, S. K., \& Unruh, Y. C. 2000b, A\&A, 353, 380

Fontenla, J. M., Avrett, E. H., \& Loeser, R. 1993, ApJ, 406, 319

Foukal, P. 1992, in The Solar Cycle, ed. K. L. Harvey, ASP Conf. Ser., 27, 439

Foukal, P., \& Fowler, L. 1984, ApJ, 281, 442

Fröhlich, C. 2000, Space Sci. Rev., 94, 15

Fröhlich, C. 2003, ESA, SP-535, 183

Fröhlich, C., \& Finsterle, W. 2001, in Recent Insights Into the Physics of the Sun and Heliosphere - Highlights from SOHO and Other Space Missions, ed. P. Brekke, B. Fleck, \& J. B. Gurman, ASP Conf. Ser., 203, 105

Fröhlich, C., \& Lean, J. 1998, Geophys. Res. Lett., 25, 4377

Fröhlich, C., Romero, J., Roth, H., et al. 1995, Sol. Phys., 162, 101

Harvey, K. L., \& White, O. R. 1999, ApJ, 515, 812

Jones, H. P., \& Ceja, J. A. 2001, in Advanced Solar Polarimetry Theory, Observation, and Instrumentation, ed. M. Sigwarth, ASP Conf. Ser., 236, 87
Jones, H. P., Duvall Jr., T. L., Harvey, J. W., et al. 1992, Sol. Phys., 139,211

Jones, H. P., Branston, D. D., Jones, P. B., \& Wills-Davey, M. J. 2000, ApJ, 529, 1070

Jones, H. P., Branston, D. D., Jones, P. B., \& Popescu, M. D. 2003, ApJ, 589, 658

Keppens, R., \& Martínez Pillet, V. 1996, A\&A, 316, 229

Krivova, N. A., \& Solanki, S. K. 2004, A\&A, 417, 1125

Krivova, N. A., Solanki, S. K., Fligge, M., \& Unruh, Y. C. 2003, A\&A, 399, L1

Kurucz, R. L. 1991, in Stellar Atmospheres - Beyond Classical Models, ed. L. Crivellari, I. Hubeny, \& D. G. Hummer (Dordrecht: Kluwer), 441

Kurucz, R. L. 1992, Rev. Mex. Astron. Astrofis., 23, 187

Martínez Pillet, V., Lites, B. W., \& Skumanich, A. 1997, ApJ, 474, 810

Neckel, H., \& Labs, D. 1994, Sol. Phys., 153, 91

Ortiz, A., Solanki, S. K., Domingo, V., Fligge, M., \& Sanahuja, B. 2002, A\&A, 388, 1036

Scherrer, P. H., Bogart, R. S., Bush, R. I., et al. 1995, Sol. Phys., 162, 129

Severino, G., Gomez, M. T., \& Caccin, B. 1994, in Solar Surface Magnetism, ed. R. J. Rutten, \& C. J. Schrijver (Dordrecht: Kluwer), 169

Solanki, S. K. 1997, in 1st Advances in Solar Physics Euroconference: Advances in the Physics of Sunspots, ed. B. Schmieder, J. C. del Toro Iniesta, \& M. Vázquez, ASP Conf. Ser., 118, 178

Solanki, S. K. 2003, A\&AR, 11, 153

Solanki, S. K., \& Rüedi, I. 2003, A\&A, 411, 249

Solanki, S. K., \& Stenflo, J. O. 1984, A\&A, 140, 185

Thornton, C. E., \& Jones, H. P. 2002, BAAS, 34, 1243

Unruh, Y. C., Solanki, S. K., \& Fligge, M. 1999, A\&A, 345, 635

Willson, R. C. 1994, in The Sun as a Variable Star, ed. J. M. Pap, C. Fröhlich, H. S. Hudson, \& S. K. Solanki, IAU Coll., 143 (Cambridge: Cambridge Univ. Press), 54 